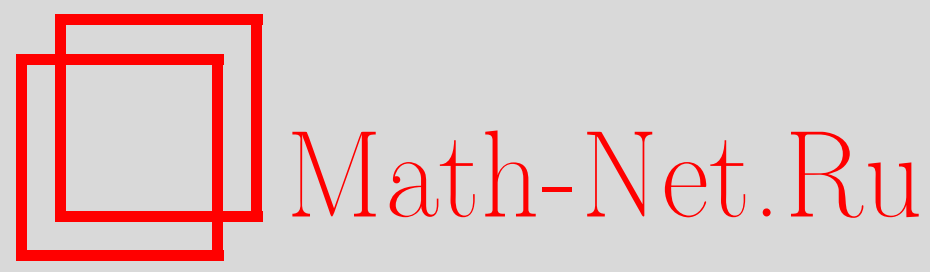

Н. Ю. Сатимов, М. Тухтасинов, О стартовом управлении системами с сосредоточенными параметрами, Матем. заметки, 2004, том 75, выпуск 5, 721-728

DOI: https://doi.org/10.4213/mzm68

Использование Общероссийского математического портала Math-Net.Ru подразумевает, что вы прочитали и согласны с пользовательским соглашением http://www.mathnet.ru/rus/agreement

Параметры загрузки:

IP : 35.173 .219 .12

26 апреля 2023 г., 14:40:38 


\title{
О СТАРТОВОМ УПРАВЛЕНИИ СИСТЕМАМИ \\ С СОСРЕДОТОЧЕННЫМИ ПАРАМЕТРАМИ
}

\author{
Н. Ю. Сатимов, М. Тухтасинов
}

\begin{abstract}
В данной работе рассматривается задача о стартовом управлении в системах обыкновенных дифференциальных уравнений. Найдены достаточные условия для разрешимости задачи в игровой постановке. Изучены структуры области управления игроков.

Библиографияя: 3 названия.
\end{abstract}

1. Рассматривается однородное линейное дифференциальное уравнение

$$
\dot{z}=A z
$$

где $z \in \mathbb{R}^{n}, n \geqslant 1 ; A$-постоянная квадратная матрица порядка $n$.

Пусть даны постоянная матрица $B$ (порядка $r \times n)$, число $T>0$ и действительнозначная векторная функция

$$
m(t)=\left(m_{1}(t), \ldots, m_{r}(t)\right)^{\prime}, \quad 0 \leqslant t \leqslant T
$$

(' - знак транспонирования).

Задача стартового управления состоит в следующем (cp. [1], [2]). Найти начальньй вектор $z^{0} \in \mathbb{R}^{n}$ так, чтобы для всех $t \in[0, T]$ имело место равенство

$$
B z(t)=m(t), \quad 0 \leqslant t \leqslant T,
$$

где $z(t), 0 \leqslant t \leqslant T,-$ решение уравнения $(1)$, удовлетворяющее условию $z(0)=z^{0}$.

Настоящая работа посвящена решению сформулированной вьше задачи стартового управления. В следующем пункте формулируется основная задача работы и доказьвается необходимый и достаточньй признак ее разрешимости (теорема 1).

В третьем пункте рассматривается игровая задача, связанная с задачей стартового управления. Получено достаточное условие ее разрешимости (теорема 2). Далее изучается дискретный аналог модельной задачи о распространении тепла в стержне, выясняется структура множества управляемости $R$ (утверждения 1,2 ). Утверждение 3 полностью решает поставленную в п. 3 игровую задачу.

В случае, когда $R$ содержит более одного элемента, решается экстремальная задача (см. (14)). В конце работы рассмотрены примеры, иллюстрирующие эффективность теоремы 2 (утверж дение 4 и следствие). 
2. Рассмотрим семейство

$$
\Sigma=\left\{B e^{t A} a, 0 \leqslant t \leqslant T: a \in \mathbb{R}^{n}\right\} .
$$

Ясно (см. [1]), что $\Sigma$ - конечномерное пространство векторных аналитических функций, определенных на $[0, T]$.

Легко заметить, что для того чтобы задача стартового управления была разрешима, необходимо и достаточно, чтобы $m(\cdot) \in \Sigma$.

Таким образом, задача стартового управления сводится к изучению структуры множества

$$
R=\left\{z^{0} \in \mathbb{R}^{n}: B e^{t A} z^{0}=m(t), 0 \leqslant t \leqslant T\right\},
$$

в частности, его одноточечности. Если множество $R$ содержит более одного элемента, то целесообразно ввести некоторый функционал, экстремум которого необходимонайти.

Введем в рассмотрение линейньй оператор $F$, действующий из $\mathbb{R}^{n}$ в $C\left([0, T], \mathbb{R}^{r}\right)$ по правилу

$$
F z^{0}=B e^{t A} z^{0}, \quad 0 \leqslant t \leqslant T \text {. }
$$

Нетрудно заметить, что одноточечность множества $R$ эквивалентна условию Ker $F=0$. В то же время справедлива следующая

Теорема 1. Для того чтобы Ker $F=\{0\}$, необходимо и достаточно выполнение условия

$$
\operatorname{rank} K=n,
$$

$2 \partial e$

$$
K=\left[\begin{array}{c}
B \\
B A \\
B A^{2} \\
\cdots \\
B A^{n-1}
\end{array}\right]
$$

ДоказАтельство. Достаточность. Пусть $\operatorname{Ker} F=\{0\}$, но $\operatorname{rank} K<n$. Тогда между столбцами матрицы $K$ существует линейная зависимость, т.е. существует ненулевой вектор $z^{0} \in \mathbb{R}^{n}$ такой, что $K z^{0}=0$. Отсюда следует, что

$$
B z^{0}=0, \quad B A z^{0}=0, \quad B A^{2} z^{0}=0, \quad \ldots, \quad B A^{n-1} z^{0}=0 .
$$

По известной теореме Гамильтона-Кэли

$$
A^{n}=\alpha_{1} A^{n-1}+\alpha_{2} A^{n-2}+\cdots+\alpha_{n} I,
$$

где $\alpha_{1}, \alpha_{2}, \ldots, \alpha_{n}$ - некоторые действительные числа.

Следовательно,

$$
B A^{n} z^{0}=\alpha_{1} B A^{n-1} z^{0}+\alpha_{2} B A^{n-2} z^{0}+\cdots+\alpha_{n} B z^{0}=0 .
$$

Из (6) легко вывести, что для всех $s=n+1, n+2, \ldots$

$$
B A^{s} z^{0}=0
$$


Значит,

$$
B e^{t A} z^{0}=B\left(I+t A+\frac{t^{2} A^{2}}{2 !}+\cdots\right) z^{0}=0
$$

для каждого $t \in[0, T]$. Из этого тождества вытекает включение $z^{0} \in \operatorname{Ker} F$, противоречащее условию $\operatorname{Ker} F=\{0\}$.

Необходимость. Пусть $\operatorname{rank} K=n$, но ядро оператора $F$ содержит ненулевой вектор $z^{0}$. Тогда для всех $t \in[0, T]$

$$
B e^{t A} z^{0} \equiv 0
$$

При $t=0$ имеем $B z^{0}=0$. Дифференцируя тождество (7) и положив $t=0$, получим равенства $B A z^{0}=0, B A^{2} z^{0}=0, \ldots, B A^{n-1} z^{0}=0$.

Следовательно, между столбцами матрищы $K$ существует линейная зависимость, означающая, что ранг матрицы $K$ меньше $n$. Мы пришли к противоречию, ибо по предположению $\operatorname{rank} K=n$. Теорема доказана.

3. Разобъем множество компонент начальных векторов $z^{0} \in \mathbb{R}^{n}$ на две части, для простоты положив

$$
z^{0}=\left(x^{0}, y^{0}\right), \quad x^{0} \in \mathbb{R}^{q}, \quad y^{0} \in \mathbb{R}^{q^{\prime}}, \quad q+q^{\prime}=n, \quad q \geqslant 1, \quad q^{\prime} \geqslant 1 .
$$

Предположим, что выбором вектора $x^{0}$ распоряжается игрок I (преследователь), выбором вектора $y^{0}-$ игрок II (преследуемьй, убегаюший).

Задача игрока I: путем выбора $x^{0}$ добиться выполнения равенства (2).

Задача игрока II: путем выбора $y^{0}$ помешать выполнению этого равенства. При выборе $x^{0}, y^{0}$ игроки располагают различной информацией. Ниже уточняется, какой информацией пользуются игроки при решении своих задач.

Пусть

$$
Q=\left\{y^{0} \in \mathbb{R}^{q^{\prime}}: \exists x^{0} \in \mathbb{R}^{q}, z^{0}=\left(x^{0}, y^{0}\right) \in R\right\} \text {. }
$$

Нетрудно убедиться в справедливости следующего утверждения.

Теорема 2. 1) Допустим, что существует вектор $x^{0} \in \mathbb{R}^{q}$ такой, что $\left\{x^{0}\right\} \times$ $\mathbb{R}^{q^{\prime}}=R$. Тогда, независимо от порядка выбора векторов $x^{0}$ и $y^{0}$ игроками I $u$ II соответственно, выигрывает игрок $\mathrm{I}$.

2) Предположим, что $Q \neq \mathbb{R}^{q^{\prime}}$. Тогда, независимо от порядка ходов, выигрывает игрок II.

3) Пусть $Q=\mathbb{R}^{q^{\prime}}$, но $\left\{x^{0}\right\} \times \mathbb{R}^{q^{\prime}}=R$ для произвольной точки $x^{0}$. Тогда, если первым делает выбор игрок I, а вторым, зная этот выбор - II, то выигрывает игрок II, а если первым делает выбор игрок II, а вторым, зная этот выбор, - I, то выигрывает игрок I (выигрыш игрока - достижение своей иели).

Приведем основные этапы докАЗАТЕЛьСтВА. 1) Игроку I достаточно выбрать $x^{0}$. Тогда для любого вектора $y^{0} \in \mathbb{R}^{q^{\prime}}$ имеем $z^{0}=\left(x^{0}, y^{0}\right) \in R$.

2) Так как по условию проекция $Q$ множества $R$ на пространства $\mathbb{R}^{q^{\prime}}$ не совпадает с $\mathbb{R}^{q^{\prime}}$, найдется $y^{0} \in \mathbb{R}^{q^{\prime}} \backslash Q$ такой, что для произвольного вектора $x^{0} \in \mathbb{R}^{q}$ имеем $z^{0}=\left(x^{0}, y^{0}\right)=R$.

3) Пусть $x^{0}$ - произвольный вектор, выбранный игроком I. По условию множество $\left\{x^{0}\right\} \times \mathbb{R}^{q^{\prime}} \in R$. Поэтому найдется вектор $y^{0} \in \mathbb{R}^{q}$ такой, что $z^{0}=\left(x^{0}, y^{0}\right)=R$. Значит, для достижения своей цели игроку II достаточно, зная $x^{0}$, выбрать $y^{0}$. 
Теперь предположим, что $x^{0}$ - произвольный вектор, выбранный игроком I. По условию $Q=\mathbb{R}^{q^{\prime}}, z^{0}=\left(x^{0}, y^{0}\right) \in R$. Следовательно, игроку I, зная $y^{0}$, достаточно выбрать $x^{0}$, чтобы достичь своей цели.

4. Рассмотрим уравнение (1), где матрица $A$ трехдиагональная; точнее, пусть

$$
A=\left(\begin{array}{cccccc}
-2 & 1 & 0 & \ldots & 0 & 0 \\
1 & -2 & 1 & \ldots & 0 & 0 \\
0 & 1 & -2 & \ldots & 0 & 0 \\
\ldots & \ldots & \ldots & \ldots & \ldots & \ldots \\
0 & 0 & 0 & \ldots & 1 & -2
\end{array}\right)
$$

Отметим, что соответствующая система уравнений получается путем равномерной аппроксимации по пространственному переменному уравнения теплопроводности стержня конечной длины, в случае, когда на концах поддерживается нулевая температура [3]. Собственные значения и векторы рассматриваемой матрищы известны [3], поэтому для любой матрищы В можно описать явно семейство функций $\Sigma$ (см. (2)).

В дальнейшем в качестве $m(\cdot)$ рассматриваются функции только из семейства $\Sigma$. Пусть матрица $B$ содержит в каждой строке только один ненулевой элемент. Если через $i_{1}, \ldots, i_{s}, s \leqslant r$, обозначить номера столбцов матрицы $B$, которые содержат ненулевой элемент, то имеет место следуюшее

УТВЕРЖДЕНИЕ 1. ДЛя того чтобы множество $R$ (см. (4)) состояло из единственного әлемента, необходимо и достаточно, чтобы $\left(i_{1}, \ldots, i_{s}, n+1\right)=1$ (здесь $\left(j_{1}, \ldots, j_{k}\right)$ означает наибольший общий делитель (НОД) чисел $\left.j_{1}, \ldots, j_{k}\right)$.

ДокАЗАТЕЛЬСтво. Необходимость. Пусть $R$ состоит из единственного элемента, но $\left(i_{1}, \ldots, i_{s}, n+1\right)=p>1$. Выбрав нетривиальное решение $z_{i}=\bar{z}_{i}(t), 0 \leqslant t \leqslant T$, $i=1, \ldots, p-1$, системы

$$
\begin{aligned}
\dot{z}_{1} & =-2 z_{1}+z_{2}, \\
\dot{z}_{2} & =z_{1}-2 z_{2}+z_{3}, \\
\ldots \ldots \ldots \ldots \ldots \ldots \ldots \ldots \ldots & \ldots \ldots \ldots \\
\dot{z}_{p-2} & =z_{p-3}-2 z_{p-2}+z_{p-1}, \\
\dot{z}_{p-1} & =z_{p-2}-2 z_{p-1},
\end{aligned}
$$

непосредственной подстановкой можно убедиться, что функция

$\bar{z}(t)=\left(\bar{z}_{1}(t), \ldots, \bar{z}_{p-1}(t), 0-\bar{z}_{p-1}(t), \ldots,-\bar{z}_{1}(t), 0, \bar{z}_{1}(t), \ldots, \bar{z}_{p-1}(t), 0, \ldots\right), \quad 0 \leqslant t \leqslant T$

является нетривиальным решением системы (1).

Пусть $z^{0}(t), 0 \leqslant t \leqslant T,-$ решение системы (1) такое, что $z^{(0)}(0) \in R$. Тогда $z(t)=z^{0}(t)+\bar{z}(t), 0 \leqslant t \leqslant T$, также является решением системы $(1)$ и

$$
B e^{t A} z(0)=m(t), \quad 0 \leqslant t \leqslant T,
$$

т.е. $z(0) \in R$, но $z(0) \neq z^{0}(0)$, что противоречит единственности элемента $R$. Значит, $\left(i_{1}, \ldots, i_{s}, n+1\right)=1$. 
Достаточность. Пусть $\left(i_{1}, \ldots, i_{s}, n+1\right)=1$, но $R$ содержит не менее двух элементов. Через $z^{\prime}(t), z^{\prime \prime}(t), 0 \leqslant t \leqslant T$, обозначим решения системы (1), соответствуюшие двум различньм элементам множества $R$ (заметим, по определению $R$ в (4) $\left.z_{i_{j}}^{\prime}(t)=z_{i_{j}}^{\prime \prime}(t)=m_{j}(t), 0 \leqslant t \leqslant T, j=1, \ldots, s\right)$.

Введем следующие обозначения:

$$
i_{0}=0, \quad i_{s+1}=n+1, \quad v(t)=z^{\prime}(t)-z^{\prime \prime}(t), \quad v_{i_{0}}(t)=v_{i_{s+1}}(t)=0, \quad 0 \leqslant t \leqslant T .
$$

Очевидно, имеем $v_{i}(t)=0,0 \leqslant t \leqslant T, i=i_{1}, \ldots, i_{s}$.

1) Пусть $n_{1}=\min \left|i_{j}-i_{k}\right|=\left|i^{\prime}-i^{\prime \prime}\right|$, где минимум берется по всем $j, k$ таким, что $j \neq k, j, k=0,1, \ldots, s+1$.

Используя уравнение (1), можно убедиться, что $v_{i+k n_{1}}(t)=0,0 \leqslant t \leqslant T, k=1,2, \ldots$, но множество этих индексов $\left\{i^{\prime}+k n_{1}\right\}$ не может содержать индексы $i_{0}, \ldots, i_{s+1}$ (естественно, здесь предполагается, что $\left.n_{1}>1\right)$, иначе $\left(i_{1}, \ldots, i_{s+1}, n+1\right)=n_{1}$-противоречие.

Переобозначим через $i_{0}, \ldots, i_{s_{1}+1}$ все индексы $i$, для которых $v_{i}(t)=0,0 \leqslant t \leqslant T$.

2) Пусть $n_{2}=\min \left|i_{j}-i_{k}\right|=\left|j^{\prime}-j^{\prime \prime}\right|$, где минимум берется по всем $j, k: j \neq k$, $j, k=0,1, \ldots, s_{1}+1$. Опять же, используя уравнение (1), можно убедиться, что $v_{j^{\prime}+k n_{2}}(t)=0, \quad 0 \leqslant t \leqslant T, \quad k=1,2, \ldots$, но эти новые индексы $\left(j^{\prime}+k n_{2}\right)$ не могут совпасть со всеми индексами $i_{0}, \ldots, i_{s_{1}+1}$ (при $\left.n_{2}>1\right)$, иначе $\left(i_{1}, \ldots, i_{s}, n+1\right)=n_{2}$.

Заметив очевидное неравенство $n_{2}<n_{1}$ и продолжив этот процесс, убедимся, что на каждом шаге итерации множество индексов вида $\left\{i_{j}\right\}$ увеличивается по крайней мере на один элемент. Поэтому через конечное число шагов получается, что $v_{i}(t)=0$, $0 \leqslant t \leqslant T, i=1, \ldots, n$, а это противоречит неединственности $R$. Утверждение 1 доказано.

Теперь займемся изучением структуры множества $R$. Пусть $R$ состоит из единственного элемента. Как найти этот элемент, зная функции $m_{1}(\cdot), \ldots, m_{s}(\cdot) ?$

Допустим, что известно решение $z=z(t), 0 \leqslant t \leqslant T$, уравнения (1), соответствующее этому элементу $\left(z_{i_{j}}(t)=m_{j}(t), 0 \leqslant t \leqslant T, j=1, \ldots, s\right)$. Подставив это решение в $(1)$, имеем тождество; не нарушая общности, примем $i_{1}<(n+1) / 2$. Тогда имеем

$$
z_{i_{1}-1}(t)+z_{i_{1}+1}(t)=\dot{m_{1}}(t)+2 m(t)=\psi_{1}(t), \quad 0 \leqslant t \leqslant T .
$$

Аналогично,

$$
z_{i_{1}-2}(t)+z_{i_{1}+2}(t)=\dot{\psi}_{1}(t)+2 \psi_{1}(t)=\psi_{2}(t), \quad 0 \leqslant t \leqslant T .
$$

Продолжив этот процесс, приходим к следующему соотношению:

$$
z_{0}(t)+z_{2 i_{1}}(t)=\dot{\psi}_{i_{1}-1}+2 \psi_{i_{1}-1}(t)=\psi_{i_{1}}(t), \quad 0 \leqslant t \leqslant T .
$$

Отсюда

$$
z_{2 i_{1}}(t)=\psi_{i_{1}}(t), \quad 0 \leqslant t \leqslant T .
$$

Аналогично, повторив описанную процедуру, имеем

$$
z_{k i_{1}}(t)=\psi_{(k-1) i_{1}}(t), \quad 0 \leqslant t \leqslant T, \quad k=1, \ldots .
$$


Отметим, что $\psi_{j}(\cdot)$ - известные функции, выражаемые с помощью функции $m_{i_{1}}(\cdot)$ и ее производных. В силу того, что числа $k i_{1}, k=0,1, \ldots$, не могут совпасть со всеми числами $i_{0}, i_{1}, \ldots, i_{s+1}$ одновременно, и используя рассуждения, которые были применены при доказательстве достаточности утверждения 1 , имеем

$$
z_{i}(t)=\psi_{i}(t), \quad 0 \leqslant t \leqslant T, \quad i=1, \ldots, n .
$$

Отсюда получаем, что

$$
\left(\psi_{1}(0), \ldots, \psi_{n}(0)\right)
$$

- единственный элемент множества $R$.

Пусть теперь множество $R$ содержит более одного элемента. Тогда из утверж дения 1 следует, что $\left(i_{1}, \ldots, i_{s}, n+1\right)=p>1$ и имеет место следующее

УТВЕРЖДЕНИЕ 2. Множество $R$ состоит из векторов, разность любых двух из которых имеет вид

$$
\left(a_{1}, \ldots, a_{p-1}, 0,-a_{p-1}, \ldots,-a_{1}, 0, a_{1}, \ldots, a_{p-1}, 0, \ldots\right)
$$

әде $a_{i}, \quad i=1, \ldots, p-1,-$ действительные числа.

ДокАЗАТЕЛЬСтво. Пусть $z^{\prime}(t), z^{\prime \prime}(t), 0 \leqslant t \leqslant T,-$ два решения уравнения $(1)$, соответствующие двум различньм элементам множества $R$. Если положить $\varphi(t)=z^{\prime}(t)-$ $z^{\prime \prime}(t), 0 \leqslant t \leqslant T$, то после подстановки в (1) легко убедиться, что $\varphi_{k p}(t)=0,0 \leqslant t \leqslant T$, $k=1, \ldots$ Кроме того, функции $\varphi_{1}(t), \ldots, \varphi_{p-1}(t), 0 \leqslant t \leqslant T$, являются решением системы (10) и удовлетворяют равенствам

$$
\begin{aligned}
\varphi_{i}(t) & =-\varphi_{2 p-i}(t)=\varphi_{2 p+i}(t)=\cdots=-\varphi_{(q-1) p-i}(t) \\
& =\varphi_{(q-1) p+i}(t), \quad 0 \leqslant t \leqslant T, \quad i=1, \ldots, p-1
\end{aligned}
$$

где $q p=n+1$, и ограничиваемся случаем, когда $q$ - нечетное число (противоположный случай рассматривается аналогично).

Из равенства (13) при $t=0$ следует, что любые два элемента множества $R$ отличаются друг от друга на элемент вида (12). Это и рассуждения, использованные при доказательстве необходимости утверж дения 1, доказьвают утверждение 2 .

Теперь займемся решением следуюшей задачи: найти

$$
\min \|a\|=\left\|a^{0}\right\|, \quad a \in R,
$$

где $\|\cdot\|$ - евклидова норма в $\mathbb{R}^{n}$.

Если $R$ содержит единственньй элемент, то поставленная задача решается очевидным образом: $a_{1}^{0}=\psi_{1}(0), \ldots, a_{n}^{0}=\psi_{n}(0)$ (см. (11)). Поэтому изучим случай, когда $R$ содержит более одного элемента. Без доказательства приводим следующее 
УТВЕРЖДЕНИЕ 3. Решением задачи (14) является

$$
\begin{aligned}
a_{k p-j}^{0} & =\frac{1}{q}\left(\sum_{i=1}^{q-1}(-1)^{i+1} i \psi_{(j-1)(q-1)+i}\right)+\sum_{i=k}^{q-1}(-1)^{i} \psi_{(j-1)(q-1)+i} \\
k & =2,4, \ldots, q-1, \quad j=1,2, \ldots, p-1 \\
a_{k p+j}^{0} & =\frac{1}{q}\left(\sum_{i=1}^{q-1}(-1)^{i+1} i \psi_{(j-1)(q-1)+i}\right)+\sum_{i=k+1}^{q-1}(-1)^{i+1} \psi_{(j-1)(q-1)+i} \\
k & =0,2,4, \ldots, q-1, \quad j=1,2, \ldots, p-1 \\
a_{k p}^{0} & =\psi_{(q-1)(p-1)+k}, \quad k=1,2, \ldots, q-1
\end{aligned}
$$

где числа $\psi_{i}, i=1,2, \ldots,(q-1) p$, находятся с помощью функиий $m_{j}(\cdot), j=1,2$, $\ldots, s$, и их производных.

Теперь, разбив управляюший параметр на две части, рассмотрим игровую задачу. При этом в зависимости от разбиения возникают разные ситуации, рассмотренные в теореме 2 .

a) Если множество $R$ состоит из единственного элемента (см. утверждение 1), то выполнено условие 2) теоремы 2. Значит, независимо от порядка ходов выигрывает игpoK II.

б) Пусть множество $R$ содержит более одного элемента (см. утверждение 2 ).

Если игрок II управляет компонентами вектора $a=\left(a_{1}, \ldots, a_{n}\right) \in R$ с соответствующими индексами, каждый из которых принадлежит множествам

$$
R_{j}=\{j, 2 p-j, 2 p+j, \ldots,(q-1) p-j,(q-1) p+j\}, \quad j=1, \ldots, p-1,
$$

(игрок I управляет остальными компонентами), то выполнено условие 3) теоремы 2.

в) Если игрок II управляет компонентами с соответствующими индексами, по меньшей мере два из которых принадлежат одному из множеств $R_{j}, j=1, \ldots, p-1$, то выполнено условие 2) теоремы 2.

5. Примеры. Пусть уравнение (1) имеет вид (ср. [1])

$$
\dot{z_{1}}=z_{2}+z_{3}, \quad \dot{z_{2}}=-a z_{2}, \quad \dot{z_{3}}=-b z_{3},
$$

где $z_{i} \in \mathbb{R}^{n}, n \geqslant 1, i=1,2,3 ; a, b$ - константы.

Пусть

$$
B=(\tilde{1}, \tilde{0}, \tilde{0})
$$

где $\tilde{1}$ - единичная квадратная матрища порядка $n$, 0 - нулевая квадратная матрища порядка $n$. Число $T$ считаем произвольным положительным, функцию $m(t), 0 \leqslant t \leqslant T,-$ принадлежащей семейству $\Sigma$ (см. (3)).

Элементарные вычисления показывают (мы их опускаем), что

$$
B z(t)=z_{10}+e_{1}(t) z_{20}+f_{1}(t) z_{30}
$$

где

$$
\left(z_{10}, z_{20}, z_{30}\right)=z^{0}, \quad e_{1}(t)=\int_{0}^{t} e^{-a s} d s, \quad f_{1}(t)=\int_{0}^{t} e^{-b s} d s
$$


Придавая векторам $z_{10}, z_{20}, z_{30}$ всевозможные значения, мы получим семейство $\Sigma$. Ясно,что каждая компонента каждого элемента $\Sigma$ есть линейная комбинация константы 1 , функций $e_{1}(\cdot)$ и $f_{1}(\cdot)$. Следовательно, и каждая компонента функции $m(\cdot)$ должна представлять одну из таких комбинаций. Если это условие вьполнено, то задача стартового управления, очевидно, разрешима для произвольной тройки $\{B, T, m(\cdot)\}$.

Далее, ясно, что начальньй вектор $z^{0}$, решающий эту задачу (при фиксированных $B, T$ и $m(\cdot))$, определяется однозначно. Следовательно, при любом порядке ходов выигрьвает игрок II.

Пусть теперь в уравнении (1) $n=2, B=(1,0), T$ - произвольное положительное число, $m(\cdot)$ - произвольный элемент $\Sigma$. Имеет место следующее

УТВЕРЖДЕНИЕ 4. 1) Пусть $A=\left\{a_{i j}\right\}, i, j=1,2, a_{12} \neq 0$. Тогда множество $R$ (см. (4)) состоит из единственного әлемента.

2) Если $a_{12}=0$, то мнохсество $R=\{m(0)\} \times \mathbb{R}^{1}$.

ДокАЗАТЕЛЬСтво. 1) Так как $z_{1}(t)=m(t), 0 \leqslant t \leqslant T$, то $\dot{m}(t)=a_{11} m(t)+a_{12} z_{2}(t)$ на $[0, T]$. Следовательно, $z_{2}(t)=\left(a_{12}\right)^{-1}\left[\dot{m}(t)-a_{11} m(t)\right]$ на $[0, T]$, т.е. компонента $z_{2}(t)$ решения $z(t)=\left(z_{1}(t), z_{2}(t)\right)$, для которого $z_{1}(t)=m(t), 0 \leqslant t \leqslant T$, определяется однозначно. Также однозначно определяется $z_{2}(0)=\left(a_{12}\right)^{-1}\left[\dot{m}(0)-a_{11} m(0)\right]$. Так как и $z_{1}(0)=m(0)$ определяется однозначно числом $m(0)$, начальньй вектор $z^{0}=\left(z_{10}, z_{20}\right)$ по функции $m(\cdot)$ определяется однозначно. Значит, $R$ - одноэлементное множество.

2) Пусть теперь $a_{12}=0$. Тогда $\dot{m}(t)=a_{11} m(t)$ и $m(t)=m(0) e^{a_{11} t}, 0 \leqslant t \leqslant T$. После подстановки функции $m(\cdot)$ в уравнение $\dot{z}_{2}=a_{21} z_{1}+a_{22} z_{2}$ получим неоднородное уравнение $\dot{z}_{2}=a_{22} z_{2}+a_{21} m(t)$, которое, очевидно, имеет бесконечное число решений, однозначно определяемых начальными значениями $z_{20}$ решения $z_{2}(t), 0 \leqslant t \leqslant T$, этого уравнения. Следовательно, $R=\{m(0)\} \times \mathbb{R}^{1}$.

СлЕДСТВИЕ. 1) Если $a_{12} \neq 0$, то независимо от порядка ходов выигрывает игрок II (теорема 2).

2) Если $a_{12}=0$, то независимо от порядка ходов выигрывает игрок I (теоремa 2).

\section{СПИСОК ЦИТИРОВАННОЙ ЛИТЕРАТУРЫ}

[1] Понтрягин Л. С. Избранные научные труды. Т. ІІ. М.: Наука, 1988.

[2] Авдонин С. А., Иванов С. А. Управляемость систем с распределенньми параметрами и семейства экспонент. Киев: УМК ВО, 1989.

[3] Самарский А. А., Николаев Е. С. Методы решения сеточных уравнений. М.: Наука, 1978.

Национальный университет республики Узбекистан, г. Ташкент

Поступило 20.06 .2001

Исправленньй вариант 13.03.2003 